\title{
Snake bite poisoning: A neglected life-threatening occupational hazard
}

\author{
Himmatrao Saluba Bawaskar
}

In India in 2005, 46,000 people died of snake bite which is one for every 2 human immunodeficiency virus/acquired immunodeficiency syndrome deaths. ${ }^{[1]}$ Still the money, energy, scientific and research time allotted to snake bite is negligible. Snake bite is a time limiting acute life-threatening medical emergency often faced by farmers, villagers, hunters, migrating population and and those in poor living conditions such as in huts and small dwelling places in the hills. Snake bite is one of the most important and neglected diseases of tropical and subtropical regions. Young earning members, active workers, farmers are the main victims. Maximum cases are reported when farmers, villagers are busy in farming. Maximum viper bites are reported during the day and early darkness in the months of November to April, although krait bite is frequent during June to November. Recently incidence of snake bite is rise because of $>12 \mathrm{~h}$ electricity load shading. ${ }^{[2]}$

The Big fours, namely, the haematotoxic (Russell's viper, Echis carinatus), and the neurotoxic (bungareus caeruleus krait and Naja naja-cobra) are the common venomous snakes that flourish all over India. However a few region-specific species such as the black cobra, green pit viper, hope nosed viper and as in the present paper of Raina et al. reported Trimeresurus albolabris (white lipped pit viper) and Gloydius himalayanus pit vipers) are reported. ${ }^{[3]}$

Clinical manifestations are almost similar except a few regional variation. Russells viper envenoming may result in neuroparalysis in the hills of Himachal Pradesh and Kochi areas. ${ }^{[3]}$ Prior to treating envenomation by Russell's viper with sufficient amount of polyvalent

From:

Bawaskar Hospital and Research Center, Mahad, Raigad, Maharashtra, India

Correspondence:

Dr. Himmatrao Saluba Bawaskar, Bawaskar Hospital and Research Center, Mahad, Raigad - 402 301, Maharashtra, India.

E-mail: himmatbawaskar@ rediffmail.com

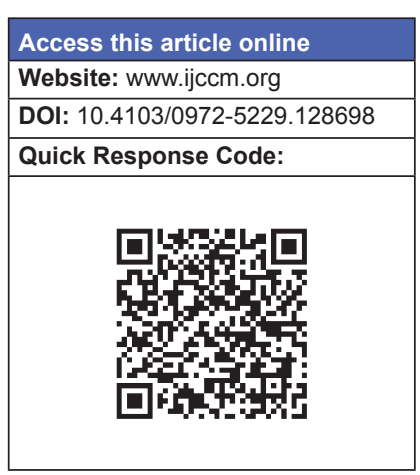

serum (10 vials) a 20 minute whole blood clotting test (20 WBCT) should be done. As the baseline test may not show coagulation abnormalities, this test should be repeated 6 hours after the last dose of the anti-snake venom (ASV). This would help decide repeat dose of ASV $(50 \mathrm{ml})$. Along with diuretics, acetyl cysteine may also help to arrest the rapid progression of swelling of the limb and subsequent amputation and renal failure due to compartment syndrome.

The E. carinatus is most aggressive and soon after a bite it rapidly runs away and many a time victims attribute the gradual swelling to usual injury which prevent the administration of ASV and such victims later go on to develop progressive swelling, compartment syndrome, septicemia, renal failure and disability. Thus farmers while walking amongst tall grass-lands or in early darkness may get rapidly progressive limb swelling with regional lymphadenopathy with or without history of snake bite in such contexts a viper bite should be diagnosed unless proved otherwise. Thus in viper bite progressive local swelling with early regional lymphadenopathy suggestive of systemic envenoming. At this time, blood may clot within $20 \mathrm{~min}$. Hypotension, shock and capillary leaks in Russell's viper is attributed bradykinin content of venom. Haemorrhage in the adrenals and the pituitary gland need to be managed with appropriate doses of methyl 
prednisolone, and fluids. In the long-term secondary amenorrhea and Sheehan's syndrome in victims of Russell's; viper have been bite reported from India. ${ }^{[4]}$ Cobra bite is often reported while handling rubbers, debris or blindly putting hand in grooves, blindly handling old objects in attics. Majority of victims die suddenly due to lethal ventricular arrhythmias from the threat of death accompanied by a surge of endogenous catecholamine is not seen in children. Sudden onset of cardiogenic shock, heart block or respiratory arrest can be revived by timely resuscitation, ASV and artificial ventilation by mouth-to mouth breathing on the spot and by bag and mask at medical centers. Venom is rich in myotoxin and phospholipids which destroy the tissues. Therefore, huge non-healing wound or ulcers may subsequently need plastic surgery or skin grafting. The authors of the present article mention early morning neuroparlysis as nothing but a victim of krait bite. ${ }^{[3,5]}$ Krait is a nocturnal snake. Its fang size is like that of an insulin needle. It enters the house during the night. While hunting rats, lizards and small kraits it may enter in to the bedding which may result in a snake bite. Due to the small size venom injected only skin deep by small fangs, there may be no signs or symptoms at the site of bite. Within $30 \mathrm{~min}$ of the bite the victims experience abdominal colic and vomiting due to release of acetyl choline and cholestokinine. The victim may experience tingling numbness or heaviness iaffecting the limb. By the time the victim reaches hospital he may manifest full-fledged neuroparalysis or locked in syndrome with absent of deep tendon reflexes. Many times krait bite victims are wrongly diagnosed as appendicitis, stroke, Guillain-Barré syndrome, hysteria, acute abdomen, botulism and undergo unnecessary surgical intervention and investigations such as brain computed tomography, magnetic resonance imaging, lumber puncture etc.

In India, there is always a short supply of ASV. Medical officers are untrained and usually have never examined or managed a snake bite case before. Envenoming is, and not the merely a history of snake bite is the indication for ASV. 20 WBCT test help re-administration of ASV. Any amount of ASV will not neutralize the venom once it is attached to neuromuscular junctions, platelets, red blood cells, renal tissues, endothelial cell, voluntary muscles (sea snake). To reduce the crisis of ASV supply medical officers should be given training regarding dose and indication of ASV. Bivalent ASV (krait + cobra) and (Echis + Russell's viper) preparation may reduce the total dose requirements. Venom detection kit may arrest the use of excessive ASV. There should be regional venom and antivenom bank for specific ASV for species of snake found in the respective regions.

Prevention is the mother of cure. Proper use of mosquito net tagged nicely to floor bedding with bamboo cot may prevent krait, scorpion, and mosquito bites alike. ${ }^{[2]}$ Thick rubber gum shoes and hand gloves may prevent viper bite. No rubble, firewood, cattle's or hens cages should be near the human dwellings. There should be routine training to medical students regarding endotracheal intubation, and examinations should include questions on snake envenomation and identification of common snakes. Primary health centers should be well-equipped with enough quantity of ASV, emergencys, endotracheal tubes, working laryngoscopes, Ambu bags and ventilators.

Snake bite is an occupational hazard. No stone should be left unturned (medical facilities, ambulances and money) to save the sons of the soil. ASV for hope nosed viper, green pit viper, sea snake is not available in India however the para specificity properties of available ASV may be helpful.

Scientists should make attempts to find alternatives to ASV as we found prazosin for the management of severe scorpion sting. ${ }^{[6]}$

\section{References}

1. Warrell DA. Snake bite: A neglected problem in twenty-first century India. Natl Med J India 2011;24:321-4.

2. Bawaskar HS, Bawaskar PH, Punde DP, Inamdar MK, Dongare RB, Bhoite RR. Profile of snakebite envenoming in rural Maharashtra, India. J Assoc Physicians India 2008;56:88-95.

3. Raina R, Raina S,Kaul R,Chander V,Jaryal A. Snakebite profile from a medical college in rural setting in the hills of Himachal Pradesh, India Indian J Crit Care Med 2014:18;130-4.

4. Uberoi HS, Achuthan AC, Kasthuri AS, Kolhe VS, Rao KR, Dugal JS. Hypopituitarism following snake bite. J Assoc Physicians India 1991;39:579-80.

5. Bawaskar HS, Bawaskar PH. Envenoming by the common krait (Bungarus caeruleus) and Asian cobra (Naja naja): Clinical manifestations and their management in a rural setting. Wilderness Environ Med 2004;15:257-66.

6. Bawaskar HS, Bawaskar PH. Call for global snake-bite control and procurement funding. Lancet 2001;357:1132-3.

How to cite this article: Bawaskar HS. Snake bite poisoning: A neglected life-threatening occupational hazard. Indian J Crit Care Med 2014;18:123-4. 